\title{
Heart Rate Variability in overweight individuals
}

\author{
Dr V .Pramodh ${ }^{1}$, Dr.M.Prashanth Kumar ${ }^{2}$, Dr.B.A. Krishna Prasad ${ }^{3}$ \\ ${ }^{1}$ Associate Professor, Department of Physiology,M.V.J.M.C\& R.H, Hoskote, Bangalore. \\ ${ }^{2}$ Professor, , Department of Physiology, R.R.M.C \&R.H,Mysore Road,Bangalore \\ ${ }^{3}$ Professor, , Department of Physiology, M.V.J.M.C\& R.H, Hoskote, Bangalore
}

\begin{abstract}
:
Aim:To assess the influence of Body Mass Index(BMI)on autonomic function using short term Heart Rate $\operatorname{Variability}(H R V)$ test.

Method: This study was conducted in 40normal weight individuals and40overweight individuals, to assess the influence of BMI on autonomic function as measured by the time domain and frequency domain parameters of short term HRV test.

Results: The HRV values were significantly reduced in overweight individuals. ( SDNN $p<0.05 ; R M S S D$ ,p<0.01;NN50 p<0.01; pNN50,p<0.01; LF, $p<0.05 ; H F, p<0.01 ; L F / H F, p<0.01)$, compared to healthy controls.

Conclusion: This study demonstrates the possibility of autonomic dysfunction in asymptomatic overweight individuals. Limitation in the present study is that the correlation of HRV with visceral fat and the influence of ethnicity on BMI were not studied. More prospective studies in this regard are required to validate this inference.
\end{abstract}

Key words: HRV, BMI, overweight, cardiovascular autonomic dysfunction.

\section{Introduction}

Increased weight gain has become a major health concern in the modern day society, probably as a byproduct of rapid industrialization and sedentary lifestyle. Presently, about 1billion peoplein the world are overweight and 475million are obese .The number has more than doubled since 1980 and 200million school-age children in the world are also overweight, making a total of nearly $1.7 \mathrm{billion}{ }^{1}$.

The Overseas Development Institute (ODI), states that, of the total 1.46 billion overweight or obese adults across the world, one in three is an Indian ${ }^{2}$.

According to WHO's The World health statistics2012 report, one in six adults obese, one in 10 diabetic and one in three has raised blood pressure ${ }^{3}$.

CAN appears to be a better predictor of major cardiac events than SMI(Silent Myocardial Ischemia). The risk linked to CAN appears to be independent of SMI and is the highest when CAN is associated with SMI ${ }^{4}$.

So, the autonomic functions can be investigated in asymptomatic overweight individuals to identify any derangements in the latent subclinical phase itself. This might help in early diagnosis and could be used prevent further complications associated with obesity.

Hence, in the present study, we conducted the HRV test, to assess the cardiac autonomic function inoverweight individuals, with BMI 27 TO 30.

\section{Materials and Methods}

Study design: 40 overweight males were enrolled in the study from the staff of a Medical college and Research Hospital, located in Bangalore rural district.

Similarly, 40 healthy male control subjects were included in the study.

These study (BMI 27 - 30) and control subjects (BMI 22-25) were chosen based on the following Inclusion and Exclusion criteria.

Inclusion Criteria:

1. Age : $45-50$ years

2. HbA1c : $<5 \%$

3. Resting heart rate: $60-80$ beats per min.

Exclusion Criteria:

1. Hypertensives.

2. Diabetics

3. Smokers and / alcoholics.

4. Cardiac complications (arrhythmias, history of Myocardial Infarction) 
5. Nephropathy (serum creatinine $>2 \mathrm{mg} / \mathrm{dl}$ )

6. Endocrine disorders (thyroid, adrenal etc)

7. Those with injuries and painful conditi0ns such as arthritis.

8. Epileptics

9. Psychiatricdisorders (depression, manic depressive illness etc)

10. Treatment with drugs like antidepressants,B blockers, antiarrhythmics, ACE inhibitors, thyroid stimulants, anti thyroid drugs.

11. Symptomatic diabetic autonomic neuropathy.

12. Trained athletes.

Ethical clearance for the study was obtained from the institutional ethical committee.

\section{Methodology:}

The procedure of the HRV test was explained to the subjects in their own language and a well informed written consent was taken.The HRV of the subjects was assessed 2 hours after food and without any caffeinated drinks or strenuous exercise meanwhile.

Height, body weight, waist circumference, and blood pressure were measured, and electrocardiogram (ECG) recordings taken. A venous blood sample was then drawn for the measurement of glucose, insulin, lipids, and glycosylated hemoglobin $(\mathrm{HbA} 1 \mathrm{c})$. Resting heart rate (HR) was determined using the calculated average time between each of the successive $\mathrm{R}$ waves over the 5-min recording.

The ECGof 5 minutes was recorded with the subjects in supine posture, in lead II, for all subjects in a state of physical and mental rest in a quiet, adequately lit and well ventilated lab.

The HRV equipment used was:RMS, VAGUS .MODEL: HRV. Serial no:HRV/121001/AOBX. Similarly, the ECG of 5 minutes was recorded in the controls. HRV values are derived by the software.

The following HRV parameters were recorded in each subject.

$\begin{array}{lll}\text { SDNN } & : & \text { Standard Deviation of the Normal to Normal beat R-R interval. } \\ \text { RMSSD } & : & \begin{array}{l}\text { Root Mean Square of the Standard Deviations of the Normal to Normal beat } \\ \text { R-R intervals. }\end{array} \\ \text { NN50 } & : & \text { Successive Normal to Normal beat R-R intervals }>50 \text { milliseconds. } \\ \text { pNN50 } & : & \begin{array}{l}\text { Proportion of Normal to Normal beat R-R intervals }>50 \text { milliseconds out of } \\ \text { Total Normal to Normal beat R-R intervals. }\end{array}\end{array}$

Fast Fourier Transform was used to assess Power Spectral components:

LF $\quad$ : $\quad$ Low Frequency $(0.04-0.1 \mathrm{~Hz})$

HF $\quad$ : High Frequency $(0.15-0.4):$ Mainly depicts the parasympathetic component

$\mathrm{LF} / \mathrm{HF} \quad$ : $\quad$ depicts the sympatho- vagal balance.

Statistical Analysis:

Student T Test was used for the statistical analysis of the results obtained.Software used statistical analysis was : Open Epi

\section{Results}

Time Domain and .Frequency Domain Parameter values are listed in Tables 1 and 2, respectively.

Table 1. Time Domain Parameters

\begin{tabular}{|l|l|l|l|}
\hline & Overweight (40) & Controls(40) & Significance \\
\hline SDNN & $45.24 \pm 7.12$ & $50.44 \pm 8.52$ & $\mathrm{p}<0.05$ \\
\hline RMSSD & $26.28 \pm 10.21$ & $35.42 \pm 10.12$ & $\mathrm{p}<0.01$ \\
\hline NN50 COUNT & $12.12 \pm 1.42$ & $18.29 \pm 3.77$ & $\mathrm{p}<0.01$ \\
\hline pNN50 & $17.71 \pm 2.87 \%$ & $28.47 \pm 09.27 \%$ & $\mathrm{p}<0.01$ \\
\hline
\end{tabular}

Table 2 .Frequency Domain Parameters

\begin{tabular}{|l|l|l|l|}
\hline & Overweight (40) & Controls(40) & Significance \\
\hline LF (n.u) & $72.8 \pm 27.37$ & $64.4 \pm 17.54$ & \\
\hline HF(n.u) & $2 \mathrm{I} .5 \pm 2.55$ & $32.72 \pm 11.99$ & $\mathrm{p}<0.05$ \\
\hline LF/HF & $4.82 \pm 1.10$ & $2.04 \pm 0.33$ & $\mathrm{p}<0.01$ \\
\hline
\end{tabular}




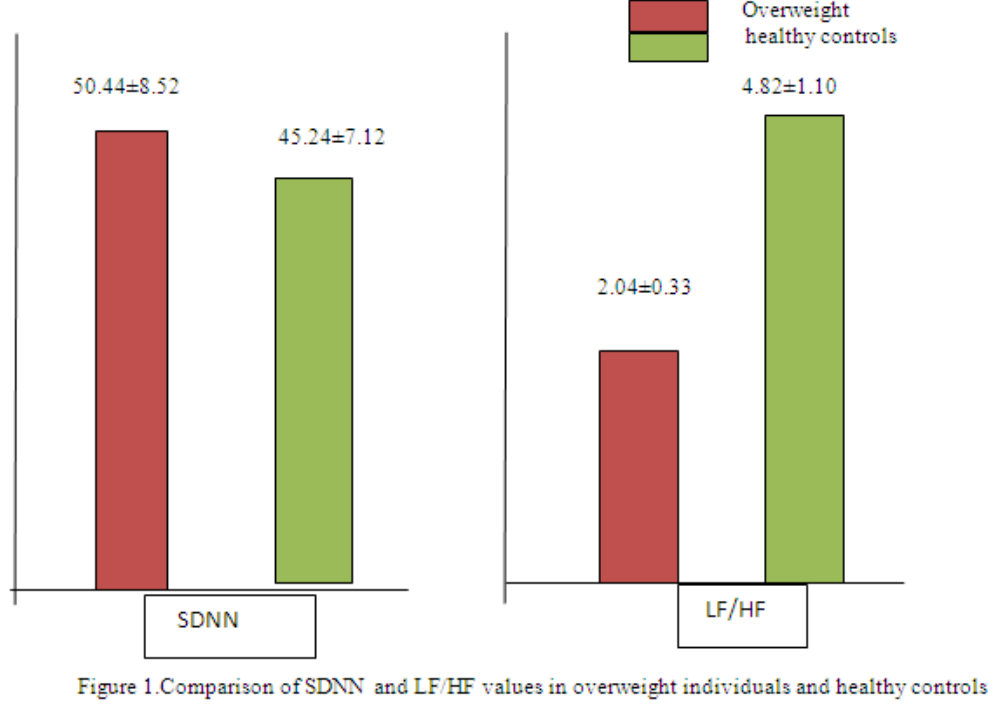

The differences in HRV values were found to be of statistical significance $(\mathrm{p}<0.01)$ between the two groups. SDNN $(\mathrm{p}<0.05)$ and LF $(\mathrm{p}<0.05)$ values in asymptomatic overweight individuals were significantly reduced as compared to that of the controls. There is also reduction in the indices signifying the parasympathetic tone such as the RMSSD ( $\mathrm{p}<0.01)$; NN50(p<0.01); pNN50(p0.01 ), HF, ( $p<0.01)$.

\section{Discussion}

Heart rate variability is oscillation in the intervals between consecutive heart beats. "Time domain" or "frequency domain methods of measuring the variation in HRV are used. The measures of HRV reflect specific physiological autonomic regulatory activities. The SDNN denotes the total power (variance) but, both RMSSD and pNN50, which calculate high frequency oscillations, reflect the parasympathetic tone.

The HF component reflects the modulation of vagus nerve discharge during respiration. The LF and VLF components reflect the more gradual co-ordination between sympathetic and parasympathetic systems, influencing the variation in R-R interval ${ }^{5}$.

Ratio of LF to HF power denotes the Sympathovagal balance. LF and LF/HF power are increased and time domain measures of HRV are decreased in upright tilt study, due to an increased sympathetic tone .Betablockade blunts these changes ${ }^{6}$.

In this study, we have observed that the $\operatorname{SDNN}(\mathrm{p}<0.05)$ and $\mathrm{LF}(\mathrm{p}<0.05)$ values in asymptomatic overweight individuals were significantly reduced as compared to that of the controls. We also found greater reduction in the indices signifying the parasympathetic tone such as the RMSSD $(\mathrm{p}<0.01)$; NN50(p<0.01); pNN50(p0.01 ), HF, ( $p<0.01)$. We also found that overweight individuals had increased LF/HF ( $<<0.01$ ) signifying altered sympathovagal balance. This might also be due to increased sympathetic tone as reflected by increased $\mathrm{LF}(\mathrm{p}<0.05)$.

Martini $G$ et al observed that24-hour and night time LF/HF ratios were significantly greater in obese. They also found an increase in heart rate and in BP, in stabilized obese normotensive children, probably due to a decrease in parasympathetic control of heart rate ${ }^{7}$.

Our study of the short term HRV of 5 minutes is effective in demonstrating the altered sympathovagal balance, probably signifying cardiovascular autonomic dysfunction in asymptomatic overweight individuals as was seen in a few long term ( 24 hour holter monitor) studies ${ }^{8}$.

Spectral analysis of HRV, using LF-to-HF ratio, is a non-invasive quantitative evaluation, efficient enough to detect the graded changes in the sympathovagal activity ${ }^{9}$.

Some of the short-term heart rate variability studies found that vagal activity was decreased in the obese group and the autonomic nervous system balance was impaired with sympathetic predominance. The LF/HF was increased significantly, and the cardiovascular autonomic balance was impaired. It is claimed that insulin resistance is the primary pathology disturbing thesympathovagalactivity ${ }^{10}$.

It has been studied by Piccirillo $\mathrm{G}$ et al that obesity diminishes modulation of heart rate but increases sympathetic modulation of arterial pressure. The obese subjects were found to have high plasma noradrenalinelevels and it was postulated that they had diminished adrenoreceptor responsiveness reflected by low LF power of heart rate ${ }^{11}$.

In one of the studies,the RMSSD, pNN50 and HF indices in milliseconds squared and SD1 of the Poincare plot, which indicate parasympathetic activity, were lower in the obese children than in the eutrophic children ${ }^{12}$. 
The study by Barbara Zahorska et al, using a tilt table test, supported the hypothesis of autonomic imbalance in obesity. They also found that HRV values improve after treatment for weight reduction for three months ${ }^{13}$.

It is also suggested that depressed parasympathetic activity in obesity is reflected in the reduced heart rate variability (HRV). A significant increase of blood pressure occurs in obese women, during handgrip. This might be due to over-reactivity of the sympathetic nervous system in them ${ }^{14}$.

Insulin resistance is suggested in obese normoglycemic subjects. Obesity may give rise to dysfunction of the peripheral autonomic nerves resembling that observed in normal-weight diabetic children and adolescents ${ }^{15}$.

Reduced sympathetic and parasympathetic activity were significantly but weakly correlated with increasing percentages of body fat. This also may promote excessive storage of energy by decreasing sympathetic activity, while defending against weight gain by decreasing parasympathetic activity. The autonomic profiling may help classifying human obesity and identifying obese patients at increased risk for cardiovascular disorders ${ }^{16}$.

It has been observed that obese patients have higher blood pressure, higher urinary norepinephrine excretion, and attenuated SDANN and HF values than lean subjects. Obese patients treated with surgery have had a mean weight loss and have displayed decreases in blood pressure and norepinephrine excretion and showed increases in SDANN and HF values. These changes were significantly greater compared to the obese control group. These findings suggest that obese patients have increased sympathetic activity and a withdrawal of vagal activity and that these autonomic disturbances improve after weight loss ${ }^{17}$.

Long ECG recordings, like 24 hour holter monitoring are useful in assessing the HRV changes in many diseased conditions such as CHF (Congestive Heart Failure) $)^{15}$.

Our study of the short term HRV of 5 minutes in diabetics is effective in demonstrating the cardiac autonomic dysfunction in asymptomatic diabetics as the other long term ( 24 hour holter monitor) studies. There are very few studies using short term HRV recordings in patients with cardiaovascular disorders and they have yielded results comparable to our study ${ }^{18}$.

Thus short term HRV test could be used as a screening tool for detecting latent cardiac autonomic dysfunction in asymptomatic overweight individuals at the clinics. This might help us to implement lifestyle modifications and institute appropriate medical intervention aimed at retarding further deterioration of autonomic functions.

Limitation in the present study is that the correlation of HRV with visceral fat and the influence of ethnicity on BMI were not studied. More prospective studies in this regard are required to validate this inference.

Hence, further studies in large overweight populations to validate these inferences are to be conducted. Then short term HRV test can be used to screen overweight individuals for CAN. Appropriate weight control and necessary medical intervention could retard the deterioration of autonomic functions associated with obesity.

\section{Conclusion}

1. Subclinical cardiac autonomic neuropathy in overweight individuals can be detected using short term HRV.

2. Sympatho-vagal balance appears to be deranged in overweight individuals, due to reduction in parasympathetic tone.

\section{Bibliography}

[1]. http://metro.co.uk/2013/04/15/nearly-1-7billion-overweight-on-fat-planet-earth-3615851/

[2]. http://timesofindia.indiatimes.com/india/Indians-adding-to-world-obesity-problem-Report/articleshow/28395856.cms

[3]. http://health.india.com/news/world-health-statistics-2012-one-in-six-adults-obese-one-in-three-hypertensiveone-in-10-diabetic/

[4]. Paul Valensi, Régis-NessimSachs,BoubakeurHarfouchePredictive Value of Cardiac Autonomic Neuropathy in Diabetic Patients With or Without Silent Myocardial Ischemia .Diabetes Care February 2001; 24 ( 2):339-343

[5]. MarekMalik ,Task Force of the European Society of Cardiology the North American Society of Pacing Electrophysiology: Heart Rate Variability Standards of Measurement, Physiological Interpretation, and Clinical Use: Circulation. 1996; 93: 1043-1065

[6]. Marc K. Lahiri, Prince J. Kannankeril, Jeffrey J. Goldberger, Assessment of Autonomic Function in Cardiovascular Disease Physiological Basis and Prognostic Implications.J Am CollCardiol. 2008; 51(18):17251733(http://content.onlinejacc.org/article.aspx?articleid=1138870)

[7]. Martini G, Riva P, Rabbia F, MoliniVHeart rate variability in childhood obesity..ClinAuton Res. 2001 Apr; 11(2):87-91.

[8]. Aronson D, Mittleman MA, Burger AJ.Measures of heart period variability as predictors of mortality in hospitalized patients with decompensated congestive heart failure..Am J Cardiol. 2004 Jan 1;93(1):59-63.

[9]. N Montano, T G Ruscone, A Porta,.Power spectrum analysis of heart rate variability to assess the changes in sympathovagal balance during graded orthostatic tilt. Circulation.1994; 90: 1826-1831

[10]. MehmetErkan Altuncu, Osman Baspinar, Mehmet KeskinThe use of short-term analysis ofheart rate variability to assess autonomic functionin obese children and its relationshipwith metabolic syndrome.Cardiol J 2012; 19, 5: 501-506.

[11]. Piccirillo G, Vetta F, Viola E, Santagada E. Heart rate and blood pressure variability in obese normotensive subjects.Int J ObesRelatMetabDisord. 1998 Aug;22(8):741-50. 
[12]. Luiz Carlos Marques Vanderlei,Carlos Marcelo Pastre,Ismael Forte FreitasJuniorAnalysis of cardiac autonomic modulation in obese and eutrophic children. Clinics (Sao Paulo).2010 August; 65(8): 789-792

[13]. BarbaraZahorska-Markiewicz, KatarzynaMizia-StecInternational Journal of Cardiology March 2003;88(1): 43-48

[14]. Zahorska-Markiewicz B, Kuagowska E, Kucio C, KlinMHeart rate variability in obesity.Int J ObesRelatMetabDisord. 1993 Jan;17(1):21-3.

[15]. Baum P, Petroff D, Classen J, Kiess W, Blüher S (2013) Dysfunction of Autonomic Nervous System in Childhood Obesity: A Cross-Sectional Study. PLoS ONE 8(1): e54546.

doi:10.1371/journal.pone.0054546(.www.plosone.org/.../info\%3Adoi\%2F10.1371\%2Fjournal.pone.0054546)

[16]. Peterson HR, Rothschild M, Weinberg CR, Fell RD, McLeish KR, Pfeifer MA. Bodyfat and the activity of the autonomic nervous system. NEngl J Med. 1988 Apr 28;318(17):1077-83.

[17]. KristjanKarason, Heart rate variability in obesity and the effect of weight loss: The American Journal of Cardiology15 April 1999 ; 83(8): Pages 1242-1247.

[18]. Migliaro ER, Contreras P.Heart rate variability: short-term studies are as useful as holter to differentiate diabetic patients from healthy subjects. Ann Noninvasive Electrocardiol. 2003 Oct;8(4):313-20 